\title{
Research on Computer Electronic Control Technology and Its Application
}

\author{
Jie $\mathrm{Yu}^{1, \mathrm{a}}$ \\ ${ }^{1}$ Changchun Vocational Institute of Technology, Changchun, Jilin, 130033 \\ ${ }^{\mathrm{a}}$ email
}

Keywords: Computer Electronic Control Technology, Application, Automatic Device

\begin{abstract}
Computer electronic control technology is a relatively complex technology, involving electronic information, automatic control and many other integrated disciplines of knowledge. Through electronic control, computer can effectively achieve the data acquisition, storage and processing, thus make the effective regulation and control of the system, so that the work efficiency can be greatly improved. Thus, computer electronic control technology has a very broad prospects and the development of all walks of life will play a very important role.
\end{abstract}

\section{Introduction}

Automatic control is mainly in the absence of manipulation, the equipment in accordance with the scheduled good procedures for self-running in the computer technology continues to promote and application of the process, the computer electronic control technology can better achieve the system of self-control and regulation. For the protection of the entire system stability and reliability of the operation has an important impact. Therefore, in the computer system to play a strong data collection, transmission, application and other functions of the case, the whole system can run more efficient and safe, is an important guarantee for automatic control system. Thus, in the information network era, the combination of computer and automatic control system and is the inevitable trend of the continuous development of society, for promoting human and economic sustainable development plays an important role. In the course of practice, the computer electronic control system mainly includes the computer hardware control, the computer software control two aspects, among them, the computer software may guarantee the system according to the hypothesis procedure movement, is reduces the accident security accident important foundation, it mainly by computer Language compilation, and computer hardware, including control objects, computers, related equipment and channels, there are many types, functions, to protect the performance of automatic control system has a great effect. At present, computer electronic control technology commonly used systems are supervisory control system, operation guidance and control system, direct digital control system and field bus control system, and people's life, work and study and other aspects have very close ties, is our country Information technology, network technology needs to attach great importance to the future development of one of the directions.

\section{Technology Status Analysis}

Computer in the industrial production process, there are many forms of interference factors, these factors cause the computer after the assembly is still a problem, and affect the normal process, can not be used normally, mainly due to the following factors.

High voltage transmission lines and electrical equipment, radio waves, lightning and so can produce electromagnetic fields, as well as objects generated by electromagnetic waves, so that the electromagnetic field in its range of space-induced sensing effect caused by spatial sensing and computer control system will interfere. The process channel of the computer can also cause interference to the computer. The common ground line set in the host and the process channel can effectively reduce the interference of the computer process channel. AC power supply network used by the computer itself is also a factor of system interference, the computer in the completion of the power supply process, the frequency of the grid will be affected by interference. Signal transmission 
process, due to the existence of the potential difference, the spread of electrical signals by the resistance of the signal transmission is slow. Relative to the propagation distance of the line, the current at a given rate of propagation process, resulting in radio waves, the incident wave and reflected wave impedance due to the terminal impedance and wave inconsistencies, so that the incident and reflected waves to reach the terminal, the reflection, reflection of the frequent, resulting in waveform and signal are affected by the pulse interference. Current wave and voltage wave transmission speed also restricts the normal operation of the computer control system.

The impact of the two major components of the computer core: the normal operation of hardware and software, to take the appropriate response to the solution. The interference of the hardware, the types and types of interference to the control system, based on different interference factors, the use of different anti-jamming methods, by excluding electromagnetic fields, process channels, radio waves and other factors, so that the computer Hardware anti-jamming effect is significantly improved.

Reduce the software interference, to interfere with the software system filtering, PC values, traps and other effective control and processing. Computer systems in the digital effective processing, the data signal transmission filter will produce interference and the computer control system for multiple signal acquisition can weaken the impact of filtering. When the computer's CPU time is damaged, PC value will be greatly affected by the manual input command NOP way, when the program fails, the instruction can be timely and accurate adjustment of the correct PC value of the track, to avoid or weaken the PC Value of the interference of the software system. In addition can use the software trap non-program interval prevention, so that the CPU interrupt or reset command is forced to boot to the specified location, the effective control of the normal use of the computer.

\section{Computer Electronic Control Technology Specific Application}

Communication technology is the basic technology of electric information acquisition system. The current communication technology in the information collecting system mainly includes wireless public network communication, power line carrier communication and optical fiber communication and so on. Wireless public network communication operation costs are higher, the maintenance of timeliness is weak, poor signal in some areas, data acquisition is difficult. Power line carrier communication construction process is simple, do not need to re-wiring, the flaw is the reliability of the communication process, security is lacking. One-time investment cost of optical fiber communication technology is too high, wiring difficult.

The operation mode of the master station of the power consumption information collecting system can be divided into two kinds: centralized and distributed. In the centralized operation mode, the investment cost is low, and the operation and maintenance work is unified. However, once the failure occurs, the scope of the operation is large and the processing capacity of the system is higher. Distributed mode of operation on the enterprise information network security and reliability requirements are low, the network resources to support the supper, but the investment cost is too large. In practical application, the main station of power consumption information collecting system is mainly used to meet the daily business needs such as electricity account, electricity analysis, line loss control, electric energy metering device.

Electricity information collection system will automatically send a reminder of the main station warning and inform the electricity user timely electricity. Intelligent charge control technology, including the realization of the main station charge control technology, collecting terminal charge control technology and energy meter charge control technology.

(1) The main station electricity technology. If the two data are the same or the former is less than the latter limit, the acquisition system will automatically execute the trip command to stop the power supply to the user, and the balance of the electricity charge and the amount of trip amount will be compared. Only when the electricity users settle the related expenses can they return to normal power supply.

(2) Energy meter charge control technology. Power users to pay the situation, the parameters of data sent to the meter through the main station for storage backup. When there is a need for power 
users to monitor and control, you can calculate the remaining electricity, according to the amount of trip restrictions, the amount of warnings, such as restrictions on the different forms of warning. Only when the user pays the arrears before they can return to normal power supply.

(3) Collection terminal charge control technology. The collection terminal carries out the charge control management, prompts the charge control information and carries on the corresponding payment early warning or the tripping control.

The application of computer electronic control technology in locomotive electronic control device mainly includes the engine electronic control device, the locomotive information transmission device, the locomotive chassis electronic control device and the locomotive body electronic control device. With the popularity of the computer, data acquisition and finishing gradually by the computer detection control to perform. And computer detection control system in locomotive electronic control devices play a big role, through the computer electronic control technology can effectively ensure the comprehensive control of locomotive production.

The engine electronic control device of the locomotive includes a locomotive ignition timing control device, a fuel injection control device, a recirculation control device and an idling operation control device. Locomotive ignition time control device, the role is to ensure that the work in different environments, the engine can also be the maximum torque or the rate of the best ignition to reach the state. Fuel injection control devices mainly control the proportion of fuel and air to ensure that locomotives can effectively control the amount of waste emissions, reduce fuel consumption, so energy saving and environmental protection. The recirculation control device mainly recovers the polluted gas which is not completely burned and fully burns again so as to reduce the emission of some harmful gases in the locomotive exhaust gas. The locomotive information transmission device comprises a locomotive navigation device, a multi-channel information transmission device and a cellular mobile phone. Locomotive navigation device is mainly used to receive satellite signals, and according to the location of the locomotive, the actual trajectory, road conditions, locomotive services and other information passed to the driver. Multi-channel information transmission device is through the display, controller and other equipment work together, the external input of information received, processing and processing, to achieve full control of the locomotive. Cellular mobile phone can achieve higher reliability of communication.

Electronically controlled power steering system, four-wheel steering, cruise control, electronic control suspension, automatic transmission, anti-lock braking device and anti-skid device are also included in the chassis control system. Electronically controlled power steering system is based on locomotive driving conditions, increase locomotive steering safety and flexibility to increase the ride comfort. Four-wheel steering device to achieve steering operation is through the control of the four wheels of the locomotive to improve the locomotive stability and controllability. Cruise is to set the speed of the locomotive, so that the locomotive can automatically control the speed, can effectively reduce the driver due to prolonged driving fatigue, improve locomotive safety. Electronic control suspension device is the height of the locomotive body to automatically adjust, making the locomotive comfort and stability of the effective control. Automatic transmission is based on the actual situation of the locomotive analysis and judgments, the automatic shift operation to protect the safety of locomotives, and to achieve energy saving and environmental protection effect. Locomotive body electronic control devices include locomotive air-conditioning control devices, locomotive electronic lighting control devices, information display system, locomotive airbag control device four parts. Locomotive air conditioning control device is obtained through the sensor inside and outside the locomotive temperature and other related information, and detailed analysis, calculation and judgments, and air conditioning switch automatic control, and ensure the temperature inside the locomotive humidity, adjust the locomotive air conditioning In the wind temperature. Locomotive electronic lighting control device is through the induction of light to control the lights switch to ensure the safety of locomotive driving, to achieve good control. Information display system refers to the locomotive driver to send the locomotive time, fuel, speed and other information to help the driver to understand the situation of the locomotive itself, and its scientific and reasonable judgments to ensure the controllability of travel. Locomotive airbag 
control device is through the induction of external forces to achieve automatic airbag air filling, to avoid the driver and the steering wheel and glass collide to ensure the safety of the driver.

\section{The Development Prospect of Computer Electronic Control Technology}

As an emerging science and technology, computer electronic control technology in China has a very broad prospect for development. Computer electronic control technology is a comprehensive technology, covering the computer technology, automatic control technology, electronic information technology, and many other professional skills. With the advent of the information age, computer electronic control technology will also move towards a more intelligent, network and integrated direction of development, the technology will become more mature and the application will become more extensive. In the near future, computer electronic control technology will be widely used in agriculture, military, aviation, medicine, construction, industry and other major fields. In addition, because the computer control system itself is through the corresponding computer language to achieve automatic control, does not require more complicated circuit design, which is very suitable for promotion in various fields, will effectively improve efficiency and promote the continuous development of the industry.

\section{Conclusion}

The computer electronic control system is a comprehensive control system which is accompanied with the continuous rise and development of the modern large-scale industrial production automation. It depends on the computer technology, the network communication technology and the control technology in the new period and plays a vital role in many fields of computer participation. Therefore, the computer electronic control system which has high-performance needs the further development of computer technology. The strong support of the relevant design language and the continuous improvement of computer electronic control technology will lead to faster economic growth and promote the progress of human social civilization.

\section{References}

[1] Li Yinhua. Study on computer electronic control technology and application [J]. Henan Science and Technology, 2014, 06 (10): 56-57.

[2] Dang Jie. The principle of computer control technology and Its future development [J]. Electronic production, 2015, 8 (6): 40-41.

[3] Zhou Juan.Application of computer detection and control technology in automotive electronic control system [J]. Acta Automation \& Instrumentation, 2014, 12 (4): 85-86.

[4] Cai Zhaowen. Application of electronic systems on the automation control technology research [J]. Technological innovation and application, 2014 (08): 55-56.

[5] Zhao Bing. Analysis of computer remote control technology and the corresponding software application [J]. Heilongjiang Science and Technology Information, 2013 (30): 101-102. 